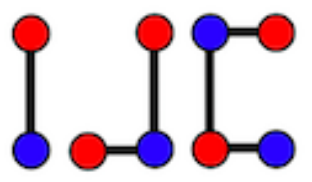

\title{
Zagreb indices of block-edge transformation graphs and their complements
}

\author{
Bommanahal Basavanagoud $^{\text {a }}$, Shreekant Patil ${ }^{\mathrm{a}}$ \\ ${ }^{a}$ Department of Mathematics, Karnatak University, Dharwad - 580 003, Karnataka, India \\ b.basavanagoud@gmail.com, shreekantpati1949@gmail.com
}

\begin{abstract}
In this paper, we obtain expressions for first and second Zagreb indices and coindices of blockedge transformation graphs $G^{a b}$. Analogous expressions are obtained also for the complements of $G^{a b}$.

Keywords: Zagreb index, Zagreb coindex, reformulated Zagreb index, reformulated Zagreb coindex, block-edge transformation graphs $G^{a b}$

Mathematics Subject Classification : 05C07

DOI: $10.19184 /$ ijc.2017.1.2.3
\end{abstract}

\section{Introduction}

Chemical graph theory is a branch of mathematics which combines graph theory and chemistry. Graph theory is used to model molecules mathematically in order to gain insight into the physical properties of these chemical compounds. The basic idea of chemical graph theory is that physico-chemical properties of molecules can be studied by using the information encoded in their corresponding chemical graphs.

In the chemical literature, there have been a few earlier attempts to shift from ordinary chemical graph to their transforms. The line graphs and the iterated line graphs were used in $[16,17,19,27]$. Attempts to use graph complements were recently reported [25].

A graph invariant is any function on a graph that does not depend on a labeling of its vertices. Such quantities are called topological indices. Zagreb indices belongs among the best investigated topological indices, but their properties and chemical applications were always studied for case

\footnotetext{
Received: 04 Oct 2016, Revised: 21 May 2017, Accepted: 12 Jun 2017.
} 
of ordinary chemical graphs [7, 12, 13, 15, 24]. Recently, some authors focused their attention to the Zagreb indices (coindices) of certain transformation graphs [2, 4, 5, 14, 21]. The present work is the continuation of research along the same lines, and is concerned with additional types of transformation graphs.

Let $G=(V, E)$ be a simple (molecular) graph. The number of vertices and edges of $G$ are denoted by $n$ and $m$ respectively. As usual $n$ is said to be order and $m$ the size of $G$. A graph of order $n$ and size $m$ will be, for short, referred to as an $(n, m)$-graph. The degree of a vertex $v \in V(G)$ is the number of vertices adjacent to $v$ in $G$. It will be denoted by $d_{G}(v)$. If $u$ and $v$ are two adjacent vertices of $G$, then the edge connecting them will be denoted by $u v$. The degree of an edge $e=u v$ in $G$, denoted by $d_{G}(e)$, is defined by $d_{G}(e)=d_{G}(u)+d_{G}(v)-2$. The complement of the graph $G$, denoted by $\bar{G}$, is the graph with vertex set $V(G)$, in which two vertices are adjacent if and only if they are not adjacent in $G$. The line graph $L(G)$ of $G$ is the graph whose vertex set is $E(G)$ in which two vertices are adjacent if and only if they are adjacent in $G$. The jump graph $J(G)$ of $G$ is the graph whose vertex set is $E(G)$ in which two vertices are adjacent if and only if they are not adjacent in $G$ [6]. A block of a graph is connected nontrivial graph having no cutvertices. For terminology not defined here we refer the reader to [20].

In this paper, we are concerned with two degree-based invariants, called first Zagreb index $M_{1}$ and second Zagreb index $M_{2}$. These are defined as

$$
M_{1}(G)=\sum_{v \in V(G)} d_{G}(v)^{2} \text { and } M_{2}(G)=\sum_{u v \in E(G)} d_{G}(u) d_{G}(v)
$$

respectively. Their mathematical theory is nowadays well elaborated. For details, see the papers [7, 14, 15, 24]. For historical data on the Zagreb indices see [13]. For surveys on degree-based topological indices see $[10,12]$.

The first Zagreb index can be written also as $[8,9]$

$$
M_{1}(G)=\sum_{u v \in E(G)}\left[d_{G}(u)+d_{G}(v)\right] .
$$

Noticing that contribution of nonadjacent vertex pairs should be taken into account when computing the weighted Wiener polynomials of certain composite graphs (see [8]) defined first Zagreb coindex and second Zagreb coindex as

$$
\bar{M}_{1}(G)=\sum_{u v \notin E(G)}\left[d_{G}(u)+d_{G}(v)\right] \text { and } \bar{M}_{2}(G)=\sum_{u v \notin E(G)} d_{G}(u) d_{G}(v)
$$

respectively.

The vertex-degree-based graph invariant

$$
F(G)=\sum_{v \in V(G)} d_{G}(v)^{3}=\sum_{u v \in E(G)}\left[d_{G}(u)^{2}+d_{G}(v)^{2}\right]
$$

was encountered in [18]. Recently there has been some interest to $F$, called "forgotten topological index"[11].

Milićević et al. [23] reformulated the Zagreb indices in terms of edge-degrees instead of vertexdegrees. The first and second reformulated Zagreb indices are defined, respectively, as 


$$
E M_{1}(G)=\sum_{e \in E(G)} d_{G}(e)^{2}=\sum_{e \sim f \in E(G)}\left[d_{G}(e)+d_{G}(f)\right] \text { and } E M_{2}(G)=\sum_{e \sim f \in E(G)} d_{G}(e) d_{G}(f) .
$$

In [22], Hosamani and Trinajstić are recently defined the first and second reformulated Zagreb coindices, respectively, as

$$
\overline{E M_{1}}(G)=\sum_{e \nsim f \in E(G)}\left[d_{G}(e)+d_{G}(f)\right] \text { and } \overline{E M_{2}}(G)=\sum_{e \nsim f \in E(G)} d_{G}(e) d_{G}(f),
$$

where $e \sim f(e \nsim f)$ means that the edges $e$ and $f$ are adjacent (not adjacent) in $G$.

The following theorems are useful for proving our results.

Theorem 1.1. $[14,26]$ For any graph $G$ of order $n$ and size $m$,

$$
M_{1}(\bar{G})=M_{1}(G)+n(n-1)^{2}-4 m(n-1) .
$$

Theorem 1.2. [1, 14] Let $G$ be any graph of order $n$ and size $m$. Then

$$
M_{1}(G)+\bar{M}_{1}(G)=2 m(n-1) .
$$

Theorem 1.3. [1, 14] Let $G$ be a simple graph. Then

$$
\bar{M}_{1}(G)=\bar{M}_{1}(\bar{G}) .
$$

Theorem 1.4. [14] Let $G$ be a graph of order $n$ and size $m$. Then

$$
\begin{aligned}
& M_{2}(\bar{G})=\frac{1}{2} n(n-1)^{3}-3 m(n-1)^{2}+2 m^{2}+\left(\frac{2 n-3}{2}\right) M_{1}(G)-M_{2}(G) \\
& \overline{M_{2}}(G)=2 m^{2}-\frac{1}{2} M_{1}(G)-M_{2}(G) \\
& \overline{M_{2}}(\bar{G})=m(n-1)^{2}-(n-1) M_{1}(G)+M_{2}(G) .
\end{aligned}
$$

\section{Block-edge transformation graphs $G^{a b}$}

Let $G=(V, E)$ be a graph with block set $U(G)=\left\{B_{i} ; B_{i}\right.$ is a block of $\left.G, 1 \leq i \leq r\right\}$. If a block $B \in U(G)$ with the edge set $\left\{e_{1}, e_{2}, \ldots, e_{s} ; s \geq 1\right\}$, then we say that the edge $e_{i}$ and block $B$ are incident with each other, where $1 \leq i \leq s$. In [3], we introduced the block-edge transformation graphs $G^{a b}$ and defined as follows:

Definition: Let $a, b$ be two variables taking values + or - . The block-edge transformation graph $G^{a b}$ is a graph whose vertex set is $E(G) \cup U(G)$, and two vertices $x$ and $y$ of $G^{a b}$ are joined by an edge if and only if one of the following holds:

(i) $x, y \in E(G) . x$ and $y$ are adjacent in $G$ if $a=+; x$ and $y$ are not adjacent in $G$ if $a=-$.

(ii) $x \in E(G), y \in U(G) . x$ and $y$ are incident with each other in $G$ if $b=+; x$ and $y$ are not incident with each other in $G$ if $b=-$. 
Thus, we obtain four kinds of block-edge transformation graphs $G^{++}, G^{+-}, G^{-+}$and $G^{--}$. The degree of a block $B$ in $G$, denoted by $d_{G}(B)$, is the number of edges incident with $B$ in $G$. We denote sum of squares of the degrees of the blocks of $G$ by $\bar{\xi}(G)$, that is $\bar{\xi}(G)=\sum_{i=1}^{r} d_{G}\left(B_{i}\right)^{2}$. The vertex $e^{\prime}\left(B^{\prime}\right)$ of $G^{a b}$ corresponding to edge $e$ (resp., block $B$ ) of $G$ and is referred as edge (resp., block)-vertex. In Figure 1 self-explanatory examples of these block-edge transformation graphs are depicted.
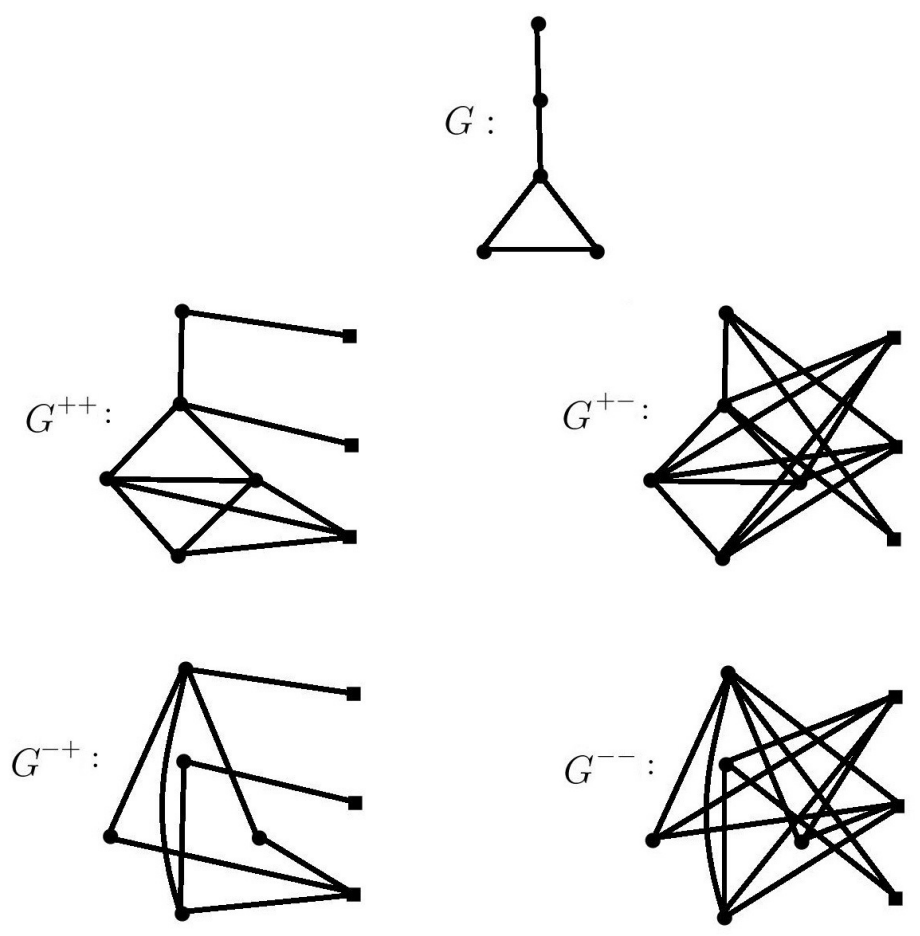

Figure 1. Graph $G$ and its block-edge transformation graphs $G^{a b}$. The edge-vertices are indicated by circles and block-vertices by squares in $G^{a b}$.

\section{Results}

Before our discussion, we introduce the following propositions, which are useful to our main results.

Proposition 3.1. Let $G$ be an $(n, m)$-graph with $r$ blocks. Then the degree of edge-vertex $e^{\prime}$ $(e=u v$ in $G)$ and block-vertex $B^{\prime}$ in $G^{a b}$ are

(i) $d_{G^{++}}\left(e^{\prime}\right)=d_{G}(u)+d_{G}(v)-1$ and $d_{G^{++}}\left(B^{\prime}\right)=d_{G}(B)$.

(ii) $d_{G^{+-}}\left(e^{\prime}\right)=d_{G}(u)+d_{G}(v)+r-3$ and $d_{G^{+-}}\left(B^{\prime}\right)=m-d_{G}(B)$.

(iii) $d_{G^{-+}}\left(e^{\prime}\right)=m+2-d_{G}(u)-d_{G}(v)$ and $d_{G^{-+}}\left(B^{\prime}\right)=d_{G}(B)$.

(iv) $d_{G^{--}}\left(e^{\prime}\right)=m+r-d_{G}(u)-d_{G}(v)$ and $d_{G^{--}}\left(B^{\prime}\right)=m-d_{G}(B)$. 
Proof. (i) $d_{G^{++}}\left(e^{\prime}\right)=d_{G}(e)+1=d_{G}(u)+d_{G}(v)-1$ and $d_{G^{++}}\left(B^{\prime}\right)=$ Number of edges incident with block $B$ in $G=d_{G}(B)$.

(ii) $d_{G^{+-}}\left(e^{\prime}\right)=d_{G}(e)+r-1=d_{G}(u)+d_{G}(v)+r-3$ and $d_{G^{+-}}\left(B^{\prime}\right)=$ Number of edges not incident with block $B$ in $G=m-d_{G}(B)$.

(iii) $d_{G^{-+}}\left(e^{\prime}\right)=m-1-d_{G}(e)+1=m+2-d_{G}(u)-d_{G}(v)$ and $d_{G^{-+}}\left(B^{\prime}\right)=$ Number of edges incident with block $B$ in $G=d_{G}(B)$.

(iv) $d_{G^{--}}\left(e^{\prime}\right)=m-1-d_{G}(e)+r-1=m+r-d_{G}(u)-d_{G}(v)$ and $d_{G^{--}}\left(B^{\prime}\right)=$ Number of edges not incident with block $B$ in $G=m-d_{G}(B)$.

Proposition 3.2. Let $G$ be an $(n, m)$-graph with $r$ blocks. Then the order of $G^{a b}$ is $m+r$.

(i) The size of $G^{++}=\frac{1}{2} M_{1}(G)$.

(ii) The size of $G^{+-}=\frac{1}{2} M_{1}(G)+m(r-2)$.

(iii) The size of $G^{-+}=\frac{m(m+3)}{2}-\frac{1}{2} M_{1}(G)$.

(iv) The size of $G^{--}=\frac{m(m+2 r-1)}{2}-\frac{1}{2} M_{1}(G)$.

Proof. Since $L(G)$ and $J(G)$ are induced subgraphs of $G^{+b}$ and $G^{-b}$ respectively. The number of edges in $L(G)$ [20] is $-m+\frac{1}{2} \sum_{v \in V(G)} d_{G}(u)^{2}=-m+\frac{1}{2} M_{1}(G)$, therefore the number of edges in $J(G)$ is $\frac{m(m-1)}{2}-$ size of $L(G)=\frac{m(m-1)}{2}+m-\frac{1}{2} M_{1}(G)=\frac{m(m+1)}{2}-\frac{1}{2} M_{1}(G)$. Hence,

(i) The size of $G^{++}=-m+\frac{1}{2} M_{1}(G)+m=\frac{1}{2} M_{1}(G)$.

(ii) The size of $G^{+-}=-m+\frac{1}{2} M_{1}(G)+m r-m=\frac{1}{2} M_{1}(G)+m(r-2)$.

(iii) The size of $G^{-+}=\frac{m(m+1)}{2}-\frac{1}{2} M_{1}(G)+m=\frac{m(m+3)}{2}-\frac{1}{2} M_{1}(G)$.

(iv) The size of $G^{--}=\frac{m(m+1)}{2}-\frac{1}{2} M_{1}(G)+m r-m=\frac{m(m+2 r-1)}{2}-\frac{1}{2} M_{1}(G)$.

Theorem 3.1. Let $G$ be an $(n, m)$-graph with $r$ blocks. Then

$$
M_{1}\left(G^{++}\right)=2 M_{2}(G)-2 M_{1}(G)+\bar{\xi}(G)+F(G)+m .
$$

Proof. By definition of the first Zagreb index, we have

$$
\begin{aligned}
M_{1}\left(G^{++}\right) & =\sum_{x \in V\left(G^{++}\right)} d_{G^{++}}(x)^{2} \\
& =\sum_{e^{\prime} \in V\left(G^{++}\right) \cap E(G)} d_{G^{++}}\left(e^{\prime}\right)^{2}+\sum_{B^{\prime} \in V\left(G^{++}\right) \cap U(G)} d_{G^{++}}\left(B^{\prime}\right)^{2} .
\end{aligned}
$$

From Proposition 3.1, we have

$$
\begin{aligned}
M_{1}\left(G^{++}\right) & =\sum_{u v \in E(G)}\left[d_{G}(u)+d_{G}(v)-1\right]^{2}+\sum_{B \in U(G)} d_{G}(B)^{2} \\
& =\sum_{u v \in E(G)}\left[d_{G}(u)+d_{G}(v)\right]^{2}+\sum_{u v \in E(G)} 1-2\left[\sum_{u v \in E(G)}\left[d_{G}(u)+d_{G}(v)\right]+\bar{\xi}(G)\right.
\end{aligned}
$$




$$
\begin{aligned}
= & \sum_{u v \in E(G)}\left[d_{G}(u)^{2}+d_{G}(v)^{2}\right]+2\left[\sum_{u v \in E(G)}\left[d_{G}(u) d_{G}(v)\right]+\sum_{u v \in E(G)} 1\right. \\
& -2\left[\sum_{u v \in E(G)}\left[d_{G}(u)+d_{G}(v)\right]\right]+\bar{\xi}(G) \\
= & F(G)+2 M_{2}(G)+m-2 M_{1}(G)+\bar{\xi}(G) .
\end{aligned}
$$

Corollary 3.1. Let $G$ be an (n, $m)$-graph with $r$ blocks. Then

$$
M_{1}\left(\overline{G^{++}}\right)=2 M_{2}(G)-2(m+r) M_{1}(G)+\bar{\xi}(G)+F(G)+(m+r)(m+r-1)^{2}+m .
$$

Proof. From Theorem 1.1, $M_{1}\left(\overline{G^{++}}\right)=M_{1}\left(G^{++}\right)+n_{1}\left(n_{1}-1\right)^{2}-4 m_{1}\left(n_{1}-1\right)$, where $n_{1}$ and $m_{1}$ are number of vertices and edges in $G^{++}$respectively. Eq. (1) follows now from Proposition 3.2 and Theorem 3.1.

Corollary 3.2. Let $G$ be an $(n, m)$-graph with $r$ blocks. Then

$$
\bar{M}_{1}\left(G^{++}\right)=(m+r+1) M_{1}(G)-2 M_{2}(G)-\bar{\xi}(G)-F(G)-m .
$$

Proof. From Theorem 1.2, $\bar{M}_{1}\left(G^{++}\right)=2 m_{1}\left(n_{1}-1\right)-M_{1}\left(G^{++}\right)$, where $n_{1}$ and $m_{1}$ are number of vertices and edges in $G^{++}$respectively. Eq. (2) follows now from Proposition 3.2 and Theorem 3.1 .

Corollary 3.3. Let $G$ be an $(n, m)$-graph with $r$ blocks. Then

$$
\bar{M}_{1}\left(\overline{G^{++}}\right)=(m+r+1) M_{1}(G)-2 M_{2}(G)-\bar{\xi}(G)-F(G)-m .
$$

Proof. Apply Theorem 1.3 and Corollary 3.2.

Theorem 3.2. Let $G$ be an $(n, m)$-graph with $r$ blocks. Then

$$
M_{1}\left(G^{+-}\right)=2 M_{2}(G)+2(r-3) M_{1}(G)+\bar{\xi}(G)+F(G)+m^{2}(r-2)+m(r-3)^{2} .
$$

Proof. Using the definition of the first Zagreb index, we have

$$
\begin{aligned}
M_{1}\left(G^{+-}\right) & =\sum_{x \in V\left(G^{+-}\right)} d_{G^{+-}}(x)^{2} \\
& =\sum_{e^{\prime} \in V\left(G^{+-}\right) \cap E(G)} d_{G^{+-}}\left(e^{\prime}\right)^{2}+\sum_{B^{\prime} \in V\left(G^{+-}\right) \cap U(G)} d_{G^{+-}}\left(B^{\prime}\right)^{2} .
\end{aligned}
$$


From Proposition 3.1, we have

$$
\begin{aligned}
M_{1}\left(G^{+-}\right)= & \sum_{u v \in E(G)}\left[d_{G}(u)+d_{G}(v)+r-3\right]^{2}+\sum_{B \in U(G)}\left(m-d_{G}(B)\right)^{2} \\
= & \sum_{u v \in E(G)}\left[d_{G}(u)+d_{G}(v)\right]^{2}+\sum_{u v \in E(G)}(r-3)^{2}+\sum_{u v \in E(G)}\left[2(r-3)\left(d_{G}(u)+d_{G}(v)\right)\right] \\
& +\sum_{B \in U(G)} m^{2}+\sum_{B \in U(G)} d_{G}(B)^{2}-\sum_{B \in U(G)} 2 m d_{G}(B) \\
= & 2 M_{2}(G)+F(G)+m(r-3)^{2}+2(r-3) M_{1}(G)+r m^{2}+\bar{\xi}(G)-2 m^{2} .
\end{aligned}
$$

Corollary 3.4. Let $G$ be an $(n, m)$-graph with $r$ blocks. Then

$$
\begin{aligned}
M_{1}\left(\overline{G^{+-}}\right)= & 2 M_{2}(G)-2(m+2) M_{1}(G)+\bar{\xi}(G)+F(G)+m^{2}(r-2)+m(r-3)^{2} \\
& +(m+r-1)[(m+r)(m+r-1)-4 m(r-2)] .
\end{aligned}
$$

Proof. From Theorem 1.1, $M_{1}\left(\overline{G^{+-}}\right)=M_{1}\left(G^{+-}\right)+n_{1}\left(n_{1}-1\right)^{2}-4 m_{1}\left(n_{1}-1\right)$, where $n_{1}$ and $m_{1}$ are number of vertices and edges in $G^{+-}$respectively. Eq. (3) follows now from Proposition 3.2 and Theorem 3.2.

Corollary 3.5. Let $G$ be an $(n, m)$-graph with $r$ blocks. Then

$$
\begin{aligned}
\bar{M}_{1}\left(G^{+-}\right)= & (m-r+5) M_{1}(G)-2 M_{2}(G)-\bar{\xi}(G)-F(G)-m^{2}(r-2)-m(r-3)^{2} \\
& +2 m(r-2)(m+r-1) .
\end{aligned}
$$

Proof. From Theorem 1.2, $\bar{M}_{1}\left(G^{+-}\right)=2 m_{1}\left(n_{1}-1\right)-M_{1}\left(G^{+-}\right)$, where $n_{1}$ and $m_{1}$ are number of vertices and edges in $G^{+-}$respectively. Eq. (4) follows now from Proposition 3.2 and Theorem 3.2 .

Corollary 3.6. Let $G$ be an $(n, m)$-graph with $r$ blocks. Then

$$
\begin{aligned}
\bar{M}_{1}\left(\overline{G^{+-}}\right)= & (m-r+5) M_{1}(G)-2 M_{2}(G)-\bar{\xi}(G)-F(G)-m^{2}(r-2)-m(r-3)^{2} \\
& +2 m(r-2)(m+r-1) .
\end{aligned}
$$

Proof. Apply Theorem 1.3 and Corollary 3.5.

Theorem 3.3. Let $G$ be an $(n, m)$-graph with $r$ blocks. Then

$$
M_{1}\left(G^{-+}\right)=2 M_{2}(G)-2(m+2) M_{1}(G)+\bar{\xi}(G)+F(G)+m(m+2)^{2} .
$$

Proof. By definition of the first Zagreb index, we have

$$
\begin{aligned}
M_{1}\left(G^{-+}\right) & =\sum_{x \in V\left(G^{-+}\right)} d_{G^{-+}}(x)^{2} \\
& =\sum_{e^{\prime} \in V\left(G^{-+}\right) \cap E(G)} d_{G^{-+}}\left(e^{\prime}\right)^{2}+\sum_{B^{\prime} \in V\left(G^{-+}\right) \cap U(G)} d_{G^{-+}}\left(B^{\prime}\right)^{2} .
\end{aligned}
$$


From Proposition 3.1, we have

$$
\begin{aligned}
M_{1}\left(G^{-+}\right)= & \sum_{u v \in E(G)}\left[m+2-d_{G}(u)-d_{G}(v)\right]^{2}+\sum_{B \in U(G)} d_{G}(B)^{2} \\
= & \sum_{u v \in E(G)}\left[d_{G}(u)+d_{G}(v)\right]^{2}+\sum_{u v \in E(G)}(m+2)^{2}-\sum_{u v \in E(G)}\left[2(m+2)\left(d_{G}(u)+d_{G}(v)\right)\right] \\
& +\bar{\xi}(G) \\
= & 2 M_{2}(G)+F(G)+m(m+2)^{2}-2(m+2) M_{1}(G)+\bar{\xi}(G) .
\end{aligned}
$$

Corollary 3.7. Let $G$ be an $(n, m)$-graph with $r$ blocks. Then

$$
\begin{aligned}
M_{1}\left(\overline{G^{-+}}\right)= & 2(r-3) M_{1}(G)+2 M_{2}(G)+\bar{\xi}(G)+F(G)+m(m+2)^{2} \\
& +(m+r-1)[(m+r)(m+r-1)-2 m(m+3)] .
\end{aligned}
$$

Proof. From Theorem 1.1, $M_{1}\left(\overline{G^{-+}}\right)=M_{1}\left(G^{-+}\right)+n_{1}\left(n_{1}-1\right)^{2}-4 m_{1}\left(n_{1}-1\right)$, where $n_{1}$ and $m_{1}$ are number of vertices and edges in $G^{-+}$respectively. Eq. (5) follows now from Proposition 3.2 and Theorem 3.3 .

Corollary 3.8. Let $G$ be an $(n, m)$-graph with $r$ blocks. Then

$$
\bar{M}_{1}\left(G^{-+}\right)=(m-r+5) M_{1}(G)-2 M_{2}(G)-\bar{\xi}(G)-F(G)-m(m+2)^{2}+m(m+3)(m+r-1) .
$$

Proof. From Theorem 1.2, $\bar{M}_{1}\left(G^{-+}\right)=2 m_{1}\left(n_{1}-1\right)-M_{1}\left(G^{-+}\right)$, where $n_{1}$ and $m_{1}$ are number of vertices and edges in $G^{-+}$respectively. Eq. (6) follows now from Proposition 3.2 and Theorem 3.3 .

Corollary 3.9. Let $G$ be an $(n, m)$-graph with $r$ blocks. Then

$\bar{M}_{1}\left(\overline{G^{-+}}\right)=(m-r+5) M_{1}(G)-2 M_{2}(G)-\bar{\xi}(G)-F(G)-m(m+2)^{2}+m(m+3)(m+r-1)$.

Proof. Apply Theorem 1.3 and Corollary 3.8.

Theorem 3.4. Let $G$ be an $(n, m)$-graph with $r$ blocks. Then

$$
M_{1}\left(G^{--}\right)=2 M_{2}(G)-2(m+r) M_{1}(G)+\bar{\xi}(G)+F(G)+m^{2}(r-2)+m(m+r)^{2} .
$$

Proof. Using the definition of the first Zagreb index, we have

$$
\begin{aligned}
M_{1}\left(G^{--}\right) & =\sum_{x \in V\left(G^{--}\right)} d_{G^{--}}(x)^{2} \\
& =\sum_{e^{\prime} \in V\left(G^{--}\right) \cap E(G)} d_{G^{--}}\left(e^{\prime}\right)^{2}+\sum_{B^{\prime} \in V\left(G^{--}\right) \cap U(G)} d_{G^{--}}\left(B^{\prime}\right)^{2} .
\end{aligned}
$$


From Proposition 3.1, we have

$$
\begin{aligned}
M_{1}\left(G^{--}\right)= & \sum_{u v \in E(G)}\left[m+r-d_{G}(u)-d_{G}(v)\right]^{2}+\sum_{B \in U(G)}\left(m-d_{G}(B)\right)^{2} \\
= & \sum_{u v \in E(G)}\left[d_{G}(u)+d_{G}(v)\right]^{2}+\sum_{u v \in E(G)}(m+r)^{2}-\sum_{u v \in E(G)}\left[2(m+r)\left(d_{G}(u)+d_{G}(v)\right)\right] \\
& +\sum_{B \in U(G)} m^{2}+\sum_{B \in U(G)} d_{G}(B)^{2}-\sum_{B \in U(G)} 2 m d_{G}(B) \\
= & 2 M_{2}(G)+F(G)+m(m+r)^{2}-2(m+r) M_{1}(G)+r m^{2}+\bar{\xi}(G)+2 m^{2} .
\end{aligned}
$$

Corollary 3.10. Let $G$ be an $(n, m)$-graph with $r$ blocks. Then

$$
\begin{aligned}
M_{1}\left(\overline{G^{--}}\right)= & 2 M_{2}(G)-2 M_{1}(G)+\bar{\xi}(G)+F(G)+m^{2}(r-2)+m(m+r)^{2} \\
& +(m+r-1)[(m+r)(m+r-1)-2 m(m+2 r-1)] .
\end{aligned}
$$

Proof. From Theorem 1.1, $M_{1}\left(\overline{G^{--}}\right)=M_{1}\left(G^{--}\right)+n_{1}\left(n_{1}-1\right)^{2}-4 m_{1}\left(n_{1}-1\right)$, where $n_{1}$ and $m_{1}$ are number of vertices and edges in $G^{--}$respectively. Eq. (7) follows now from Proposition 3.2 and Theorem 3.4.

Corollary 3.11. Let $G$ be an $(n, m)$-graph with $r$ blocks. Then

$\bar{M}_{1}\left(G^{--}\right)=(m+r+1) M_{1}(G)-2 M_{2}(G)-\bar{\xi}(G)-F(G)-m^{2}(r-2)-m(m+r)^{2}+m(m+2 r-1)(m+r-1)$.

Proof. From Theorem 1.2, $\bar{M}_{1}\left(G^{--}\right)=2 m_{1}\left(n_{1}-1\right)-M_{1}\left(G^{--}\right)$, where $n_{1}$ and $m_{1}$ are number of vertices and edges in $G^{--}$respectively. Eq. (8) follows now from Proposition 3.2 and Theorem 3.4 .

Corollary 3.12. Let $G$ be an $(n, m)$-graph with $r$ blocks. Then

$$
\begin{aligned}
\bar{M}_{1}\left(\overline{G^{--}}\right)= & (m+r+1) M_{1}(G)-2 M_{2}(G)-\bar{\xi}(G)-F(G)-m^{2}(r-2)-m(m+r)^{2} \\
& +m(m+2 r-1)(m+r-1) .
\end{aligned}
$$

Proof. Apply Theorem 1.3 and Corollary 3.11.

From Theorem 1.4, it is clear that if $M_{1}\left(G^{a b}\right)$ and $M_{2}\left(G^{a b}\right)$ are known then $M_{2}\left(\overline{G^{a b}}\right), \overline{M_{2}}\left(G^{a b}\right)$ and $\overline{M_{2}}\left(\overline{G^{a b}}\right)$ are known, what really needs to be calculated are expressions for $M_{2}\left(G^{a b}\right)$.

In order to determine the expression for second Zagreb index of $G^{a b}$, we need to introduce two auxiliary degree-based indices.

Let $G$ be a (molecular) graph. Denote an edge $e(=u v$ in $G)$ is incident (not incident) with a block $B$ by $e \sim B(e \nsim B)$. Then we define two auxiliary indices as follows:

$$
M B(G)=\sum_{e \sim B}\left[d_{G}(u)+d_{G}(v)\right] d_{G}(B) \text { and } \overline{M B}(G)=\sum_{e \nsim B}\left[d_{G}(u)+d_{G}(v)\right] d_{G}(B) .
$$


Theorem 3.5. Let $G$ be an $(n, m)$-graph with $r$ blocks. Then $M_{2}\left(G^{++}\right)=E M_{1}(G)+E M_{2}(G)+\frac{1}{2} M_{1}(G)+M B(G)-m-\bar{\xi}(G)$.

Proof. By definition of the second Zagreb index, we have

$$
\begin{aligned}
M_{2}\left(G^{++}\right) & =\sum_{x y \in E\left(G^{++}\right)} d_{G^{++}}(x) d_{G^{++}}(y) \\
& =\sum_{e^{\prime} f^{\prime} \in E\left(G^{++}\right) \cap E(L(G))} d_{G^{++}}\left(e^{\prime}\right) d_{G^{++}}\left(f^{\prime}\right)+\sum_{e^{\prime} B^{\prime} \in E\left(G^{++}\right) \backslash E(L(G))} d_{G^{++}}\left(e^{\prime}\right) d_{G^{++}}\left(B^{\prime}\right) .
\end{aligned}
$$

From Proposition 3.1, we have

$$
\begin{aligned}
M_{2}\left(G^{++}\right) & =\sum_{e=u v \sim f=v w}\left[d_{G}(u)+d_{G}(v)-1\right]\left[d_{G}(v)+d_{G}(w)-1\right]+\sum_{e \sim B}\left[d_{G}(u)+d_{G}(v)-1\right] d_{G}(B) \\
& =\sum_{e \sim f}\left[d_{G}(e)+1\right]\left[d_{G}(f)+1\right]+\sum_{e \sim B}\left[\left(d_{G}(u)+d_{G}(v)\right) d_{G}(B)-d_{G}(B)\right] \\
& =\sum_{e \sim f}\left[d_{G}(e)+d_{G}(f)\right]+\sum_{e \sim f}\left[d_{G}(e) d_{G}(f)\right]+\sum_{e \sim f} 1+\sum_{e \sim B}\left[\left(d_{G}(u)+d_{G}(v)\right) d_{G}(B)\right]-\sum_{e \sim B} d_{G}(B) .
\end{aligned}
$$

$\sum_{e \sim B} d_{G}(B)=\sum_{B \in U(G)} d_{G}(B)^{2}=\bar{\xi}(G)$ as the quantity $d_{G}(B)$ appears $d_{G}(B)$ times. Hence,

$$
M_{2}\left(G^{++}\right)=E M_{1}(G)+E M_{2}(G)-m+\frac{1}{2} M_{1}(G)+M B(G)-\bar{\xi}(G) .
$$

Theorem 3.6. Let $G$ be an $(n, m)$-graph with $r$ blocks. Then

$$
\begin{aligned}
M_{2}\left(G^{+-}\right)= & (r-1) E M_{1}(G)+E M_{2}(G)+\frac{(r-1)^{2}}{2} M_{1}(G)-\overline{M B}(G)-m(r-1)^{2} \\
& +m^{2}(r-3)(r-1)+m(r-1) M_{1}(G)-(r-3)\left(m^{2}-\bar{\xi}(G)\right) .
\end{aligned}
$$

Proof. Using the definition of the second Zagreb index, we have

$$
\begin{aligned}
M_{2}\left(G^{+-}\right) & =\sum_{x y \in E\left(G^{+-}\right)} d_{G^{+-}}(x) d_{G^{+-}}(y) \\
& =\sum_{e^{\prime} f^{\prime} \in E\left(G^{+-}\right) \cap E(L(G))} d_{G^{+-}}\left(e^{\prime}\right) d_{G^{+-}}\left(f^{\prime}\right)+\sum_{e^{\prime} B^{\prime} \in E\left(G^{+-}\right) \backslash E(L(G))} d_{G^{+-}}\left(e^{\prime}\right) d_{G^{+-}}\left(B^{\prime}\right) .
\end{aligned}
$$

From Proposition 3.1, we have

$$
\begin{aligned}
M_{2}\left(G^{+-}\right)= & \sum_{e=u v \sim f=v w}\left[d_{G}(u)+d_{G}(v)+r-3\right]\left[d_{G}(v)+d_{G}(w)+r-3\right] \\
& +\sum_{e \nsim B}\left[d_{G}(u)+d_{G}(v)+r-3\right]\left[m-d_{G}(B)\right] \\
= & \sum_{e \sim f}\left[d_{G}(e)+r-1\right]\left[d_{G}(f)+r-1\right] \\
& +\sum_{e \nsim B}\left[m\left(d_{G}(u)+d_{G}(v)\right)-\left(d_{G}(u)+d_{G}(v)\right) d_{G}(B)+(r-3) m-(r-3) d_{G}(B)\right] .
\end{aligned}
$$


$\sum_{e \nsim B} d_{G}(B)=\sum_{B \in U(G)}\left(m-d_{G}(B)\right) d_{G}(B)=m^{2}-\bar{\xi}(G)$ as the quantity $d_{G}(B)$ appears $m-d_{G}(B)$ times. Hence,

$$
\begin{aligned}
M_{2}\left(G^{+-}\right)= & (r-1) E M_{1}(G)+E M_{2}(G)+(r-1)^{2}\left[-m+\frac{1}{2} M_{1}(G)\right] \\
& +m(r-1) M_{1}(G)-\overline{M B}(G)+m^{2}(r-3)(r-1)-(r-3)\left(m^{2}-\bar{\xi}(G)\right) .
\end{aligned}
$$

Theorem 3.7. Let $G$ be an $(n, m)$-graph with $r$ blocks. Then

$M_{2}\left(G^{-+}\right)=\overline{E M}_{2}(G)-m \overline{E M}_{1}(G)-\frac{m^{2}}{2} M_{1}(G)-M B(G)+\frac{m^{3}(m+1)}{2}+(m+2) \bar{\xi}(G)$.

Proof. By definition of the second Zagreb index, we have

$$
\begin{aligned}
M_{2}\left(G^{-+}\right) & =\sum_{x y \in E\left(G^{-+}\right)} d_{G^{-+}}(x) d_{G^{-+}}(y) \\
& =\sum_{e^{\prime} f^{\prime} \in E\left(G^{-+}\right) \cap E(J(G))} d_{G^{-+}}\left(e^{\prime}\right) d_{G^{-+}}\left(f^{\prime}\right)+\sum_{e^{\prime} B^{\prime} \in E\left(G^{-+}\right) \backslash E(J(G))} d_{G^{-+}}\left(e^{\prime}\right) d_{G^{-+}}\left(B^{\prime}\right) .
\end{aligned}
$$

From Proposition 3.1, we have

$$
\begin{aligned}
M_{2}\left(G^{-+}\right)= & \sum_{e=u v \nsim f=w z}\left[m+2-d_{G}(u)-d_{G}(v)\right]\left[m+2-d_{G}(w)-d_{G}(z)\right] \\
& +\sum_{e \sim B}\left[m+2-d_{G}(u)-d_{G}(v)\right] d_{G}(B) \\
= & \sum_{e \nsim f}\left[m-d_{G}(e)\right]\left[m-d_{G}(f)\right]+\sum_{e \sim B}\left[(m+2) d_{G}(B)-\left(d_{G}(u)+d_{G}(v)\right) d_{G}(B)\right] \\
= & \overline{E M}_{2}(G)-m \overline{E M}_{1}(G)+m^{2}\left[\frac{m(m-1)}{2}+m-\frac{1}{2} M_{1}(G)\right]+(m+2) \bar{\xi}(G)-M B(G) .
\end{aligned}
$$

Theorem 3.8. Let $G$ be an $(n, m)$-graph with $r$ blocks. Then

$$
\begin{aligned}
M_{2}\left(G^{--}\right)= & \overline{E M}_{2}(G)-(m+r-2) \overline{E M}_{1}(G)-\frac{(m+r-2)^{2}}{2} M_{1}(G)+\overline{M B}(G) \\
& +\frac{m(m+1)(m+r-2)^{2}}{2}+m^{2}(m+r)(r-1)-m(r-1) M_{1}(G)-(m+r)\left(m^{2}-\bar{\xi}(G)\right) .
\end{aligned}
$$

Proof. Using the definition of the second Zagreb index, we have

$$
\begin{aligned}
M_{2}\left(G^{--}\right) & =\sum_{x y \in E\left(G^{--}\right)} d_{G^{--}}(x) d_{G^{--}}(y) \\
& =\sum_{e^{\prime} f^{\prime} \in E\left(G^{--}\right) \cap E(J(G))} d_{G^{--}}\left(e^{\prime}\right) d_{G^{--}}\left(f^{\prime}\right)+\sum_{e^{\prime} B^{\prime} \in E\left(G^{--}\right) \backslash E(J(G))} d_{G^{--}}\left(e^{\prime}\right) d_{G^{--}}\left(B^{\prime}\right) .
\end{aligned}
$$


From Proposition 3.1, we have

$$
\begin{aligned}
& M_{2}\left(G^{--}\right)=\sum_{e=u v \nsim f=w z}\left[m+r-d_{G}(u)-d_{G}(v)\right]\left[m+r-d_{G}(w)-d_{G}(z)\right] \\
& +\sum_{e \nsim B}\left[m+r-d_{G}(u)-d_{G}(v)\right]\left[m-d_{G}(B)\right] \\
& =\sum_{e \nsim f}\left[m+r-2-d_{G}(e)\right]\left[m+r-2-d_{G}(f)\right] \\
& +\sum_{e \nsim B}\left[\left(d_{G}(u)+d_{G}(v)\right) d_{G}(B)-m\left(d_{G}(u)+d_{G}(v)\right)+m(m+r)-(m+r) d_{G}(B)\right] \\
& =\overline{E M}_{2}(G)-(m+r-2) \overline{E M}_{1}(G)+(m+r-2)^{2}\left[\frac{m(m-1)}{2}+m-\frac{1}{2} M_{1}(G)\right] \\
& +\overline{M B}(G)-m(r-1) M_{1}(G)+m^{2}(m+r)(r-1)-(m+r)\left(m^{2}-\bar{\xi}(G)\right) .
\end{aligned}
$$

\section{Acknowledgement}

The authors would like to thank the referee for his/her careful reading and useful suggestions which led us to improve the paper.

${ }^{a}$ This research was supported by UGC-SAP DRS-III, New Delhi, India for 2016-2021: F.510/3/DRSIII/2016(SAP-I) Dated: $29^{t} h$ Feb. 2016.

\section{References}

[1] A. R. Ashrafi, T. Došli $\breve{c}$, A. Hamzeh, The Zagreb coindices of graph operations, Discr. Appl. Math. 158 (2010) 1571-1578.

[2] B. Basavanagoud, S. Patil, Multiplicative Zagreb indices and coindices of some derived graphs, Opuscula Math. 36(3) (2016) 287-299.

[3] B. Basavanagoud, S. Patil, On the block-edge transformation graphs $G^{a b}$, International Research Journal of Pure Algebra 5(5) (2015) 75-80.

[4] B. Basavanagoud, I. Gutman, V. R. Desai, Zagreb indices of generalized transformation graphs and their complements, Kragujevac J. Sci. 37 (2015) 99-112.

[5] B. Basavanagoud, I. Gutman, C. S. Gali, On second Zagreb index and coindex of some derived graphs, Kragujevac J. Sci. 37 (2015) 113-121.

[6] G. Chartrand, H. Hevia, E. B. Jarette, M. Schultz, Subgraph distance in graphs defined by edge transfers, Discrete Math., 170 (1997) 63-79.

[7] K. C. Das, I. Gutman, Some properties of the second Zagreb index, MATCH Commun. Math. Comput. Chem. 52 (2004) 103-112. 
[8] T. Došličc, Vertex-weighted Wiener polynomials for composite graphs, Ars Math. Contemp. 1 (2008) 66-80.

[9] T. Došlič , B. Furtula, A. Graovac, I. Gutman, S. Moradi, Z. Yarahmadi, On vertex-degreebased molecular structure descriptors, MATCH Commun. Math. Comput. Chem. 66 (2011) $613-626$.

[10] B. Furtula, I. Gutman, M. Dehmer, On structure-sensitivity of degree-based topological indices, Appl. Math. Comput. 219 (2013) 8973-8978.

[11] B. Furtula, I. Gutman, A forgotten topological index, J. Math. Chem. 53 (2015) 1184-1190.

[12] I. Gutman, Degree-based topological indices, Croat. Chem. Acta 86 (2013) 351-361.

[13] I. Gutman, On the origin of two degree-based topological indices, Bull. Acad. Serbe Sci. Arts (Cl. Sci. Math. Natur.) 146 (2014) 39-52.

[14] I. Gutman, B. Furtula, Z. Kovijanić Vukićević, G. Popivoda, Zagreb indices and coindices, MATCH Commun. Math. Comput. Chem. 74 (2015) 5-16.

[15] I. Gutman, K. C. Das, The first Zagreb index 30 years after, MATCH Commun. Math. Comput. Chem. 50 (2004) 83-92.

[16] I. Gutman, $\check{Z}$. Tomović, On the application of line graphs in quantitative structure-property studies, J. Serb. Chem. Soc. 65 (2000) 577-580.

[17] I. Gutman, $\check{Z}$. Tomović, More on the line graph model for predicting physico-chemical properties of alkanes, ACH - Models Chem. 137 (2000) 439-445.

[18] I. Gutman, N. Trinajstić, Graph theory and molecular orbitals. Total П-electron energy of alternant hydrocarbons, Chem. Phys. Lett. 17 (1972) 535-538.

[19] I. Gutman, ŽZ. Tomović, B. K. Mishra, M. Kuanar, On the use of iterated line graphs in quantitative structure-property studies, Indian J. Chem. 40A (2001) 4-11.

[20] F. Harary, Graph Theory, Addison-Wesley, Reading, Mass 1969.

[21] S. M. Hosamani, I. Gutman, Zagreb indices of transformation graphs and total transformation graphs, Appl. Math. Comput. 247 (2014) 1156-1160.

[22] S. M. Hosamani, N. Trinajstić, On reformulated Zagreb coindices, Research Gate 2015-05-08 T 09:07:00 UTC.

[23] A. Milićević, S. Nikolić, N. Trinajstić, On reformulated Zagreb indices, Mol. Divers. 8 (2004) 393-399.

[24] S. Nikolić, G. Kovačević, A. Miličević, N. Trinajstić, The Zagreb indices 30 years after, Croat. Chem. Acta 76 (2003) 113-124. 
[25] J. Senbagamalar, J. Baskar Babujee, I. Gutman, On Wiener index of graph complements, Trans. Comb. 3(2) (2014) 11-15.

[26] G. Su, L. Xiong, L. Xu, The Nordhaus-Gaddum-type inequalities for the Zagreb index and coindex of graphs, Appl. Math. Lett. 25 (2012) 1701-1707.

[27] $\check{Z}$. Tomović, I. Gutman, Modeling boiling points of cycloalkanes by means of iterated line graph sequences, J. Chem. Inf. Comput. Sci. 41 (2001) 1041-1045. 